DOELLLW-239

\title{
A Comparison and Cross-Reference of Commercial Low-Level Radioactive Waste Acceptance Criteria
}

Thomas A. Kerr

April 1997

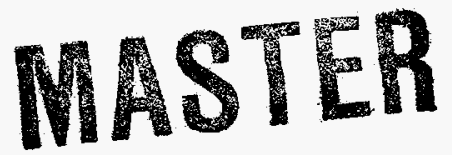

DEtriention of tris DOCuMEN is varumeo h

Prepared for the

U.S. Department of Energy

DOE Idaho Operations Office 


\section{DISCLAIMER}

This report was prepared as an account of work sponsored by an agency of the United States Government. Neither the United States Government nor any agency thereof, nor any of their employees, makes any warranty, express or implied, or assumes any legal liability or responsibility for the accuracy, completeness, or usefulness of any information, apparatus, product, or process disclosed, or represents that its use would not infringe privately owned rights. Reference herein to any specific commercial product, process, or service by trade name, trademark, manufacturer, or otherwise does not necessarily constitute or imply its endorsement, recommendation, or favoring by the United States Government or any agency thereof. The views and opinions of authors expressed herein do not necessarily state or reflect those of the United States Government or any agency thereof. 


\section{DISCLAIMER}

Portions of this document may be illegible electronic image products. Images are produced from the best available original document. 


\section{ABSTRACT}

This document, prepared by the National Low-Level Waste Management Program at the Idaho National Engineering and Environmental Laboratory, is a comparison and crossreference of commercial low-level radioactive waste acceptance criteria. Many of these are draft or preliminary criteria as well as implemented criteria at operating low-level radioactive waste management facilities. Waste acceptance criteria from the following entities are included: U. S. Nuclear Regulatory Commission, South Carolina, Washington, Utah, Nevada, California, Illinois, Texas, North Carolina, Nebraska, Pennsylvania, New York, and the Midwest Compact Region. Criteria in the matrix include the following: physical form, chemical form, liquid limits, void space in packages, concentration averaging, types of packaging, chelating agents, solidification media, stability requirements, sorptive media, gas, oil, biological waste, pyrophorics, source material, special nuclear material, package dimensions, incinerator ash, dewatered resin, transuranics, and mixed waste. Each criterion in the matrix is cross-referenced to its source document so that exact requirements can be determined. 


\section{CONTENT}

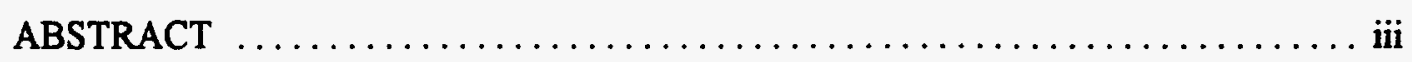

INTRODUCTION $\ldots \ldots \ldots \ldots \ldots \ldots \ldots \ldots \ldots \ldots \ldots \ldots \ldots \ldots \ldots \ldots$

Instructions for Use of Matrix $\ldots \ldots \ldots \ldots \ldots \ldots \ldots \ldots \ldots \ldots$

WASTE ACCEPTANCE CRITERIA $: \ldots \ldots \ldots \ldots \ldots \ldots \ldots \ldots \ldots \ldots \ldots \ldots \ldots \ldots \ldots \ldots \ldots$

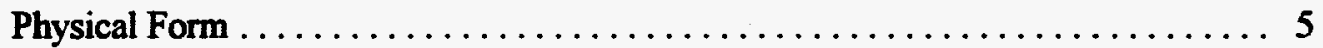

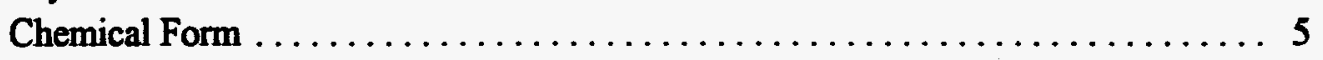

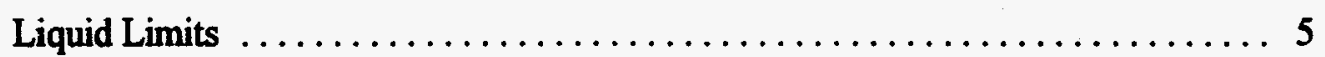

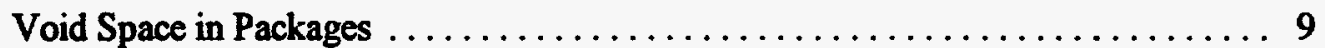

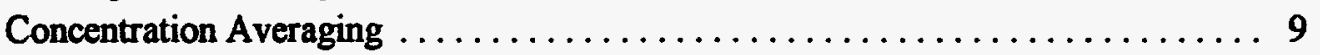

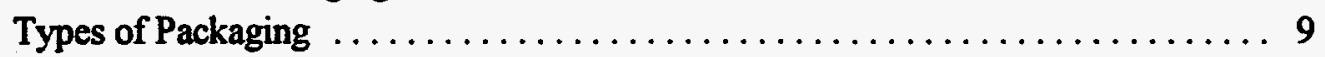

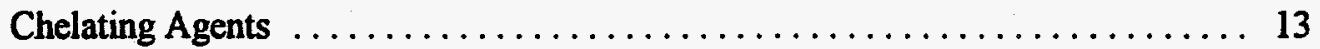

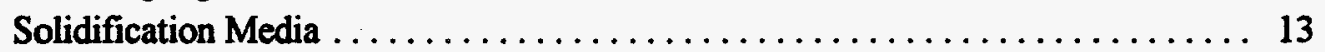

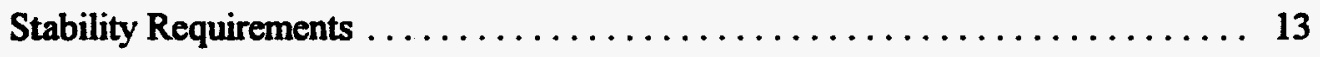

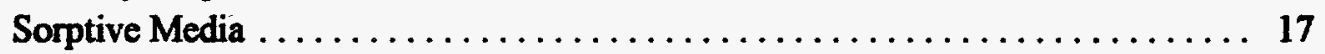

Oil ............................................ 17

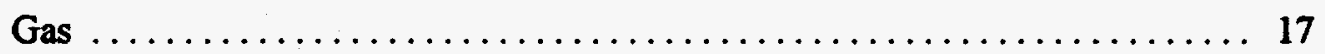

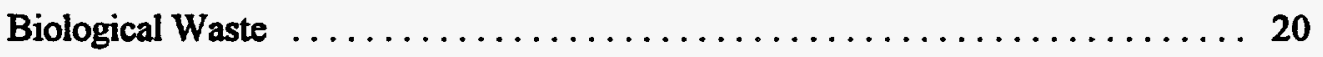

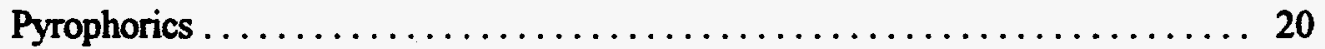

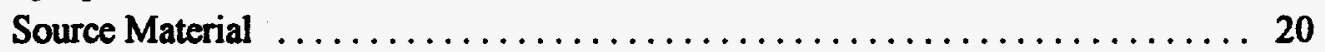

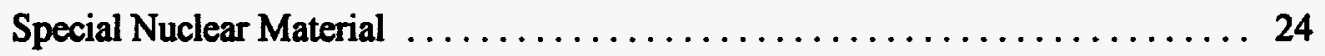

Package Dimensions . . . . . . . . . . . . . . . . . . . . . . . . 24

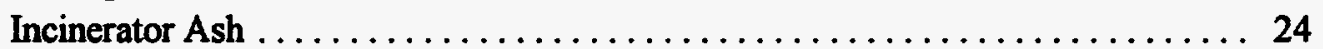

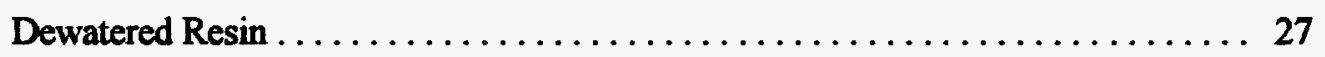

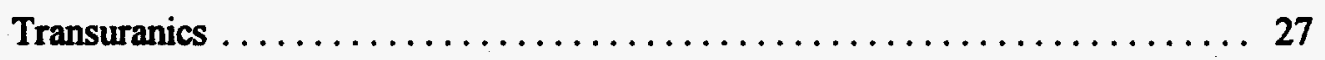

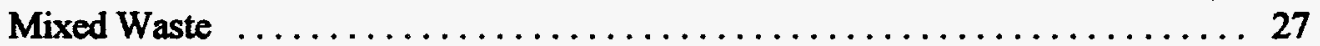




\section{INTRODUCTION}

This document is a matrix of Waste Acceptance Criteria (WAC) derived from various states, commercial facilities, regulations, and license agreements. Each section presents specific WAC (e.g., physical form, chemical form, and liquid limits) and compares them to the various states, Nuclear Regulatory Commission (NRC), Compact Region, and commercial disposal facilities' source requirements for these criteria. This comparison provides a cross-reference between each individual criterion and the applicable source requirements.

\section{Instructions for Use of Matrix}

For simplicity, each criterion in the matrix has been paraphrased. References to the appropriate source document's paragraph or condition are contained in each criterion box of the matrix below the dashed line following the paraphrased criterion. Abbreviations used in the matrix are included in the following section entitled Sources for the Commercial Low-Level Radioactive Waste Acceptance Criteria That Appear in the Matrix. For exact wording of each criterion, refer to the appropriate source document(s) for that state, agency, or entity. For ease of handling and clarity, the matrix compares 21 criteria in groups of three in the following order:

- Physical form, chemical form, liquid limits

- Void space in packages, concentration averaging, types of packaging

- Chelating agents, solidification media, stability requirements

- $\quad$ Sorptive media, oil, gas

- Biological waste, pyrophorics, source material

- Special nuclear material, package dimensions, incinerator ash

- Dewatered resin, transuranics, mixed waste. 
Sources for the Commercial Low-Level Radioactive Waste Acceptance Criteria That Appear in the Matrix:

\section{U. S. Nuclear Regulatory Commission (NRC):}

1. Title 10 of the Code of Federal Regulations, Part 61

2. Title 10 of the Code of Federal Regulations, Part 20, Appendix F

3. Branch Technical Position on Waste Form (Revision 1), January 1991 (Abbreviation Used in Matrix: BTP)

\section{South Carolina (SC):}

1. South Carolina Department of Health and Environmental Control Radioactive Material, License \#097, Amendment 46 (Abbreviation Used in Matrix: SCL)

2. U.S. Nuclear Regulatory Commission License \#12-13536-01, Amendment 26 (Abbreviation Used in Matrix: NRCL)

3. Barnwell Waste Management Facility Site Disposal Criteria (Chem-Nuclear Systems, Inc. Document \# S20-AD-010 Revision 12) (Abbreviation Used in Matrix: BSC)

\section{Washington (WA):}

1. State of Washington Radioactive Materials License \#WN-I019-2, Amendment \#18 (Abbreviation Used in Matrix: WAL)

2. U.S. Nuclear Regulatory Commission License \#16-19204-01, Amendment 11 (Abbreviation Used in Matrix: NRCL)

3. State of Washington Administrative Code 246-249-080 (for NORM)

Utah (UT):

1. State of Utah Radioactive Materials License \# UT 2300249, Amendment \#20 (Abbreviation Used in Matrix: UTL)

2. State of Utah Hazardous Waste Permit EPA Identification \#UTD982598898 (Abbreviation Used in Matrix: UTHWP) 
3. Letter dated August 31, 1995 from Vernon E. Andrews (Envirocare) to Dane Finefrock (UT Division of Radiation Control) regarding concentration averaging practices (Abbreviation Used in Matrix: LTR-08/95)

4. Reply (dated September 6, 1995) to LTR-08/95 from William Sinclair (UT Radiation Control Board) to Vernon E. Andrews (Envirocare) regarding concentration averaging practices (Abbreviation Used in Matrix: LTR-09/95)

5. Information Notice (dated May 7, 1996) from the Division of Radiation Control, Utah Department of Environmental Quality (Abbreviation Used in Matrix: UT IN-05-96)

Nevada (NV):

Nevada State Health Division Radioactive Material License \#13-11-0043-02, Amendment 13

California (CA):

California Department of Health Services Radioactive Material License \#4505-36

Illinois (IL):

Illinois Department of Nuclear Safety Notice of Proposed Rules (draft February 1991), Title 32

Chapter II Subchapter d Part 607

Texas (TX):

Texas Low-Level Radioactive Waste Disposal Authority Draft Proposed Rules, Title 31, Part XV Chapter 451 Subchapter B

North Carolina (NC):

December 1993 License Application Wake/Chatham Safety Analysis Report, Chapter 11, Revision 1, submitted by Chem-Nuclear Systems, Inc. to the North Carolina Division of Radiation Protection

Nebraska (NE):

Waste Acceptance Criteria NOP-8101-1.01, Revision 0, submitted to the Nebraska Department of Health by U.S. Ecology, Inc. (dated May 19, 1995)

Pennsylvania (PA):

Title 25 PA Code Chapter 236 
New York (NY)

1. 6NYCRR Part 382, Regulation of Low-Level Radioactive Waste (LLRW), Disposal Facilities: Certification of Proposed Sites and Disposal Methods

2. 6 NYCRR Part 383, Regulation of Low-Level Radioactive Waste (LLRW), Disposal Facilities: Design, Construction, Post-Closure, and Institutional Control

Midwest Compact Region (MW):

Preliminary Waste Acceptance Criteria (dated January 27, 1995) 
Waste Acceptance Criteria:

\author{
Physical Form \\ Chemical Form \\ Liquid Limits
}




\begin{tabular}{|c|c|c|c|}
\hline \multicolumn{4}{|c|}{ COMMERCLAL GOW-LEVEL RADIOACTIVE WA STE ACCGPTANCE CRIIERRA } \\
\hline $18 \times 8 \times$ & PHYSICAL FORM & CHEMBCAU FORM & UQUTIOIMIYS \\
\hline $\mathrm{NRC}$ & $\begin{array}{l}\text { Solid, absorbed liquid, gas } \\
61.56(a)(2) \\
61.56(a)(7)\end{array}$ & $\begin{array}{l}\text { No explosive or gaseous reactions } \\
61.56(a)(4) \\
61.56(a)(5)\end{array}$ & $\begin{array}{l}\leq 1 \% \text { vol. HIC or } \leq 0.5 \% \text { vol. for } \\
\text { other waste } \\
61.56(\mathrm{a})(3) \\
61.56(\mathrm{~b})(2)\end{array}$ \\
\hline SO & $\begin{array}{l}\text { Solid, gas } \\
\text { SCL Condition 32(A) } \\
\text { SCL Condition } 34 \\
\text { SCL Condition } 47 \\
\text { BSC } 13.3 \\
\text { BSC } 13.5\end{array}$ & $\begin{array}{l}\text { No xylene, toluene, dioxane, organic } \\
\text { scintillation liquids or other hazardous } \\
\text { organic solutions, solidified or } \\
\text { otherwise. Will accept Ecosint A, } \\
\text { Ecosint O, Opti-fluor, Ultima Gold, } \\
\text { Ultima Gold LLT, Ready-Safe, Ready } \\
\text { Cap, Ready Filter, and Meltilex. No } \\
\text { explosive or gaseous reactions } \\
\text { SCL Condition } 43 \\
\text { SCL Condition } 49 \\
\text { SCL Condition 50 } \\
\text { BSC } 13.5 .3 \\
\text { BSC } 13.7 \\
\text { BSC } 13.9 \\
\text { BSC } 13.11\end{array}$ & $\begin{array}{l}\leq 1 \% \text { vol. HIC or } \leq 0.5 \% \text { vol. for } \\
\text { other waste } \\
\text { SCL Conditions } 32 \text { (A-C) } \\
\text { BSC } 13.6\end{array}$ \\
\hline WA & $\begin{array}{l}\text { Solid, absorbed liquid, liquids (Class } \\
\text { A only not to exceed } 50 \mathrm{~mL} \text { in vials } \\
\text { used for clinical testing), gas } \\
\text { WAL Condition } 28 \\
\text { WAL Condition } 31 \\
\text { WAL Condition } 32 \\
\text { WAL Condition } 35\end{array}$ & $\begin{array}{l}\text { No explosive or gaseous reactions } \\
\text { WAL Condition } 22 \\
\text { WAL Condition } 23\end{array}$ & $\begin{array}{l}\leq 1 \% \text { vol. HIC or } \leq 0.5 \% \text { vol. for } \\
\text { other waste } \\
\text { WAL Condition } 29 \\
\text { WAL Condition } 30\end{array}$ \\
\hline U19. & $\begin{array}{l}\text { Solid (volumetric bulky soil or soil- } \\
\text { like materials or debris) } \\
\text { UTL Condition } 7 \\
\text { UTL Condition } 38 \\
\end{array}$ & $\begin{array}{l}\text { Various hazardous chemicals for } \\
\text { mixed waste } \\
\text { UTHWP }\end{array}$ & $\begin{array}{l}\text { No free liquid is acceptable } \\
\text { UTL Condition } 18\end{array}$ \\
\hline NY? & $\begin{array}{l}\text { Solid, absorbed liquid, gas } \\
\text { Condition } 21 \\
\text { Condition } 24 \\
\text { Condition } 27 \\
\text { Condition } 30 \\
\end{array}$ & $\begin{array}{l}\text { No explosive or gaseous reactions } \\
\text { Condition } 19 \\
\text { Condition } 20\end{array}$ & $\begin{array}{l}\leq 1 \% \text { vol. HIC or } \leq 0.5 \% \text { vol. for } \\
\text { other waste } \\
\text { Condition } 25 \\
\text { Condition } 26\end{array}$ \\
\hline
\end{tabular}




\begin{tabular}{|c|c|c|c|}
\hline \multicolumn{4}{|c|}{ COMMERCIAT LOW-LEVEL RADIOACTIVE WASTE ACCEPTANCE ORITERIA } \\
\hline 18X8: & PHYSICAL FORM & CHEMICAL FORM & UOUID UIMIISS \\
\hline $\mathrm{CA}^{3}$ & $\begin{array}{l}\text { Solid, gas } \\
\text { Condition } 55 \\
\text { Condition } 65\end{array}$ & $\begin{array}{l}\text { No xylene, toluene, dioxane, } \\
\text { scintillation fluids or other organic } \\
\text { liquids. No explosive or gaseous } \\
\text { reactions } \\
\text { Condition } 53 \\
\text { Condition } 54 \\
\text { Condition } 64\end{array}$ & $\begin{array}{l}\leq 1 \% \text { vol. HIC or } \leq 0.5 \% \text { vol. for } \\
\text { other waste } \\
\begin{array}{l}\text { Condition } 57 \\
\text { Condition } 58\end{array}\end{array}$ \\
\hline $11 \%$ & $\frac{\text { Solid }}{607.30(a)}$ & $\begin{array}{l}\text { No explosive or gaseous reactions } \\
607.30(\mathrm{i}) \\
607.30(\mathrm{k})\end{array}$ & $\begin{array}{l}\text { Atmospheric condensation only } \\
607.30(a)\end{array}$ \\
\hline $14 \%$ & $\begin{array}{l}\text { Solid, absorbed liquid, gas } \\
451.26(\mathrm{~b}) \\
451.26(\mathrm{c}) \\
451.26(\mathrm{e})(4)\end{array}$ & $\begin{array}{l}\text { No explosive or gaseous reactions } \\
451.26(e)(1) \\
451.26(e)(2)\end{array}$ & $\begin{array}{l}\leq 1 \% \text { vol. HIC or } \leq 0.5 \% \text { vol. for } \\
\text { stabilized waste } \\
451.26(b)\end{array}$ \\
\hline 84 & $\begin{array}{l}\text { Solid, gas } \\
236.521(3) \\
236.521(8)\end{array}$ & $\begin{array}{l}\text { No explosive or gaseous reactions } \\
236.521(5) \\
236.521(6)\end{array}$ & $\begin{array}{l}\leq 1 \% \text { vol. HIC or } \leq 0.5 \% \text { vol. for } \\
\text { other waste } \\
236.523\end{array}$ \\
\hline 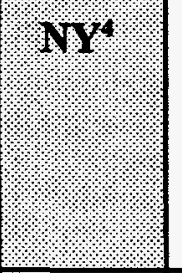 & $\frac{\text { Solid, liquid, gas }}{382.81(\mathrm{a})}$ & $\begin{array}{l}\text { No explosive or other reactions at } \\
\text { normal temp. and pressure and no } \\
\text { explosive or exothermic reactions with } \\
\text { water } \\
382.81(a)(4)\end{array}$ & $\frac{<0.5 \% \text { of waste volume }}{382.81 \text { (a) (3) }}$ \\
\hline $\mathrm{NC}^{6}$. & $\begin{array}{l}\text { Solid } \\
11.3(2) \\
11.3(5)\end{array}$ & $\begin{array}{l}\text { No explosive or other reactions at } \\
\text { normal temp. and pressure and no } \\
\text { explosive or exothermic reactions with } \\
\text { water } \\
11.3(10)\end{array}$ & $\begin{array}{l}<1 \% \text { vol. HIC or } 50.5 \% \text { vol. for } \\
\text { other waste } \\
11.3(3) \\
11.3(4)\end{array}$ \\
\hline $\mathrm{NE}^{\mathrm{s}}$ & $\begin{array}{l}\text { Solid, absorbed liquid, liquids (Class } \\
\text { A only not to exceed } 50 \mathrm{~mL} \text { in vials } \\
\text { used for clinical testing), gas } \\
8.12 \\
8.13 \\
8.17 \\
8.18 \\
8.21\end{array}$ & $\begin{array}{l}\text { No explosive or gaseous reactions } \\
8.7 \\
8.10\end{array}$ & $\begin{array}{l}\leq 1 \% \text { vol. HC or } s 0.5 \% \text { vol. for } \\
\text { other waste } \\
8.13 \\
8.14 \\
8.15\end{array}$ \\
\hline
\end{tabular}




\begin{tabular}{|c|c|c|c|}
\hline \multicolumn{4}{|c|}{ COMMERCIAL LOW-LEVEL RADIOACTIVE WASTE ACCEPTANCE CRITERIA } \\
\hline 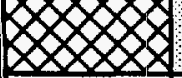 & PHYSICALFORM & CHEMICAL FORM & LIQUHD LIMIIS \\
\hline $1 \mathrm{HW}^{6}$ & $\begin{array}{l}\text { Solid, gas } \\
\mathrm{B}(7) \\
\mathrm{B}(9) \\
\mathrm{B}(10)\end{array}$ & $\begin{array}{l}\text { No explosive or gaseous reactions. } \\
B(3) \\
B(4)\end{array}$ & $\begin{array}{l}\leq 1 \% \text { vol. HIC or } \leq 0.5 \% \text { vol. for } \\
\text { other waste. Must be } \\
\text { noncorrosive, pH 4-11. } \\
\text { B (8) } \\
\text { B (11) } \\
\text { B (12) } \\
\text { B (13) }\end{array}$ \\
\hline
\end{tabular}


Waste Acceptance Criteria:

\author{
Void Space in Packages \\ Concentration Averaging \\ Types of Packaging
}




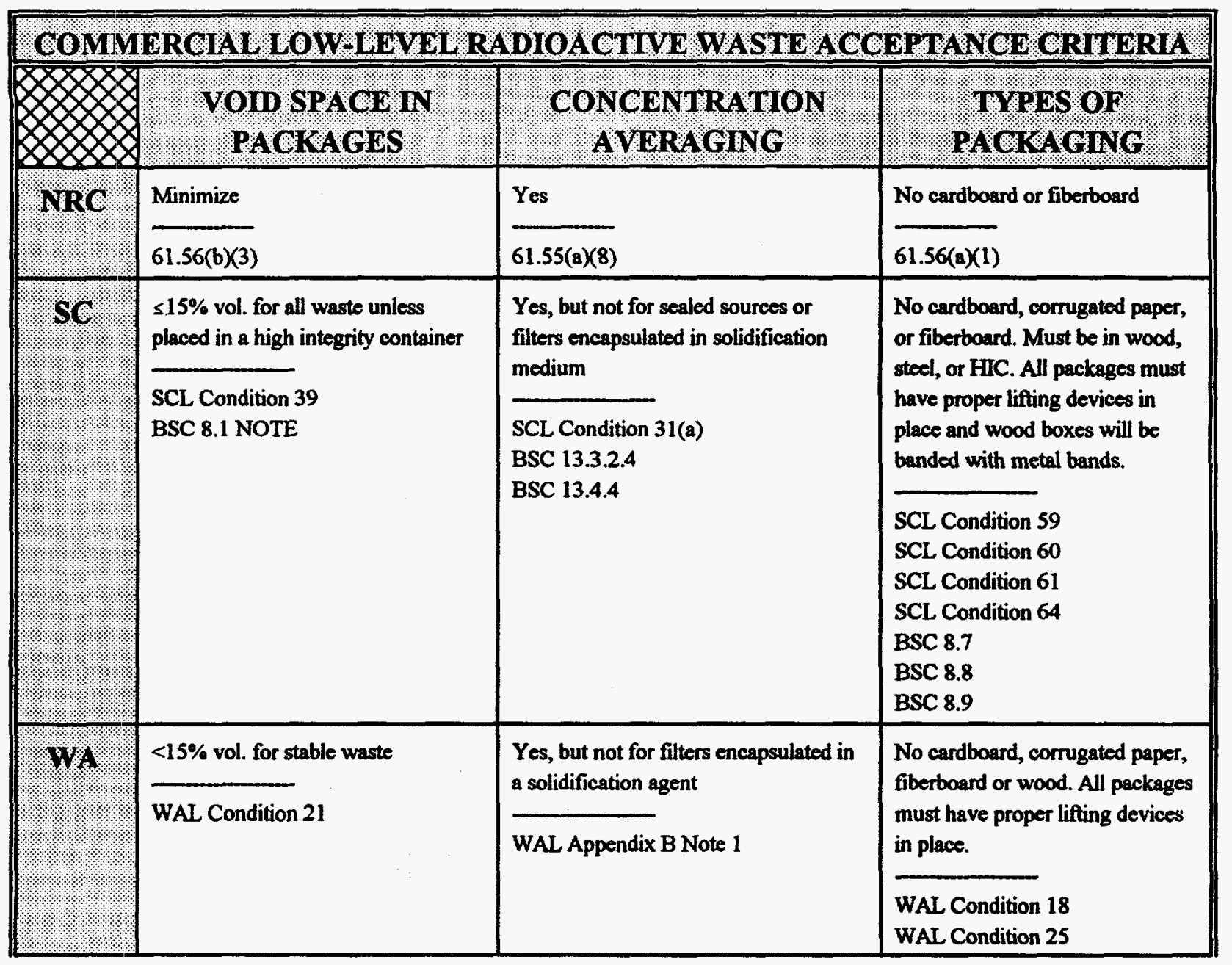




\begin{tabular}{|c|c|c|c|}
\hline \multicolumn{4}{|c|}{ COMMERCIAL LOW LEVEL RADIOACMVE WASTE ACCEPTANCE ORIIERIA } \\
\hline XXXX| & $\begin{array}{l}\text { YOD SPACE WN } \\
\text { PACKAGES }\end{array}$ & $\begin{array}{l}\text { CONCENTRA TION } \\
\text { AVERAGING }\end{array}$ & PHOR SOSO \\
\hline 10 & & $\begin{array}{l}\text { Weighted average of a shipment } \\
\text { (defined as a series of conveyances } \\
\text { from a single generator arriving within } \\
72 \text { hours) will meet license } \\
\text { concentrations; however, single } \\
\text { conveyances within the shipment may } \\
\text { be up to } 10 \text { times the license limit up } \\
\text { to the Class A limit. } \\
\text { If the weighted average of the } \\
\text { shipment exceeds the license limit, it } \\
\text { may be accepted up to } 10 \text { times the } \\
\text { license limit. It will then either be } \\
\text { blended with other waste to meet } \\
\text { license limits (for soil-like materials) or } \\
\text { clean soil, CLSM concrete, or other } \\
\text { waste (for debris). } \\
\text { UTL Condition } 8 \\
\text { UTL Condition } 14 \\
\text { UTL Condition } 16 \\
\text { UTL Condition } 38 \\
\text { LTR 08/95 }\end{array}$ & $\begin{array}{l}\text { Most waste is disposed as bulk or } \\
\text { unpackaged material; however, } \\
\text { oversized debris in the form of } \\
\text { the following filled containers } \\
\text { may be accepted: B-25 boxes, } \\
\text { B-12 boxes, standard drums of at } \\
\text { least } 50 \text { gallons, over-pack } \\
\text { drums, and other monolithic } \\
\text { forms similar in size and shape to } \\
\text { those listed. } \\
\text { UTL Condition } 37 \\
\text { UTL Condition } 38\end{array}$ \\
\hline $\mathrm{NV}^{2}$ & $\begin{array}{l}<15 \% \text { vol. for stable waste. Does } \\
\text { not apply to HICs. } \\
\text { Condition } 18\end{array}$ & $\begin{array}{l}\text { Yes, includes solidification agent wt. } \\
\text { \& vol. } \\
\text { Appendix } B(1)\end{array}$ & $\begin{array}{l}\text { No cardboard, corrugated paper, } \\
\text { fiberboard or wood } \\
\text { Condition } 15 \\
\text { Condition } 16 \\
\text { Condition } 17\end{array}$ \\
\hline $\mathrm{C}_{4} \mathrm{~s}^{2}$ & $\begin{array}{l}<15 \% \text { vol. for stable waste. Does } \\
\text { not apply to HICs. } \\
\text { Condition } 52\end{array}$ & $\begin{array}{l}\text { Yes, except for sealed sources or filters } \\
\text { encapsulated in solidification medium } \\
\text { Appendix A Note a }\end{array}$ & $\begin{array}{l}\text { No cardboard, corrugated paper, } \\
\text { fiberboard, or wood. Closed } \\
\text { metal, polyethylene, or HICs } \\
\text { only. Proper lifting devices must } \\
\text { be attached. } \\
\text { Condition } 48 \\
\text { Condition } 50 \\
\text { Condition } 51\end{array}$ \\
\hline 16 & $\frac{\text { Minimize }}{607.30(c)}$ & $\begin{array}{l}\text { Yes, except for solids encapsulated in } \\
\text { solidification medium. } \\
607.30(a)\end{array}$ & \\
\hline
\end{tabular}




\begin{tabular}{|c|c|c|c|}
\hline 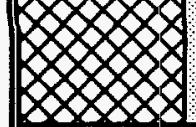 & $\begin{array}{l}\text { VOW SPACE IN } \\
\text { PACKAGES }\end{array}$ & $\begin{array}{l}\text { CONCENTRATION } \\
\text { AVERAGING }\end{array}$ & PYPR SONS \\
\hline $14 x^{4}$ & $\frac{\text { Minimize }}{451.25(h)}$ & & $\begin{array}{l}\text { No cardboard, corrugated paper, } \\
\text { wood, or fiberboard. } \\
451.25(\text { c) } \\
451.25(\text { i) }\end{array}$ \\
\hline $\mathrm{PA}$ & $\frac{\text { Minimize }}{236.524}$ & $\frac{\text { Yes }}{236.507}$ & $\begin{array}{l}\text { No cardboard, wood, or } \\
\text { fiberboard } \\
236.521\end{array}$ \\
\hline $\mathrm{M}$ & $\frac{\text { Minimize }}{382.81(b)(2)}$ & $\begin{array}{l}\text { Yes, except for sealed sources or filters } \\
\text { encapsulated in solidification medium } \\
\frac{382.80(\mathrm{~h})(2)}{}\end{array}$ & $\frac{\text { No cardboard or fiberboard }}{382.81(a)(1)}$ \\
\hline $\mathrm{NC}$ & & $\begin{array}{l}\text { Yes, includes solidification agent wt. } \\
\frac{\text { \& vol. }}{11.3(1)(a)}\end{array}$ & $\begin{array}{l}\text { No cardboard or fiberboard. } \\
\text { Radiation level cannot exceed } \\
500 \mathrm{R} / \mathrm{hr} \text { on contact. } \\
11.3(12) \\
11.5\end{array}$ \\
\hline $\mathrm{NE}^{\mathrm{S}}$ & $\frac{s 15 \text { vol. of disposal container }}{8.6}$ & & $\begin{array}{l}\text { No cardboard, corrugated paper, } \\
\text { wood, fiberboard, or combustible } \\
\text { packaging. All drums must be } \\
\text { palletized on nonwood pallets. } \\
\text { Proper lifting devices must be } \\
\text { included. } \\
8.3 .1 \\
8.4 \\
10.2 \\
11.2 \\
12.1\end{array}$ \\
\hline $11 w^{6}$ & $\frac{\text { Minimize }}{\mathrm{C}(2)}$ & & $\begin{array}{l}\text { No cardboard, corrugated paper, } \\
\text { fiberboard, or wood as outer } \\
\text { disposal container. Will accept } \\
\text { HĆs and containers approved by } \\
\text { DOT/NRC/host state. } \\
\text { C (3) } \\
\text { C (4) } \\
\text { C (5) }\end{array}$ \\
\hline
\end{tabular}


Waste Acceptance Criteria:

\author{
Chelating Agents \\ Solidification Media \\ Stability Requirements
}




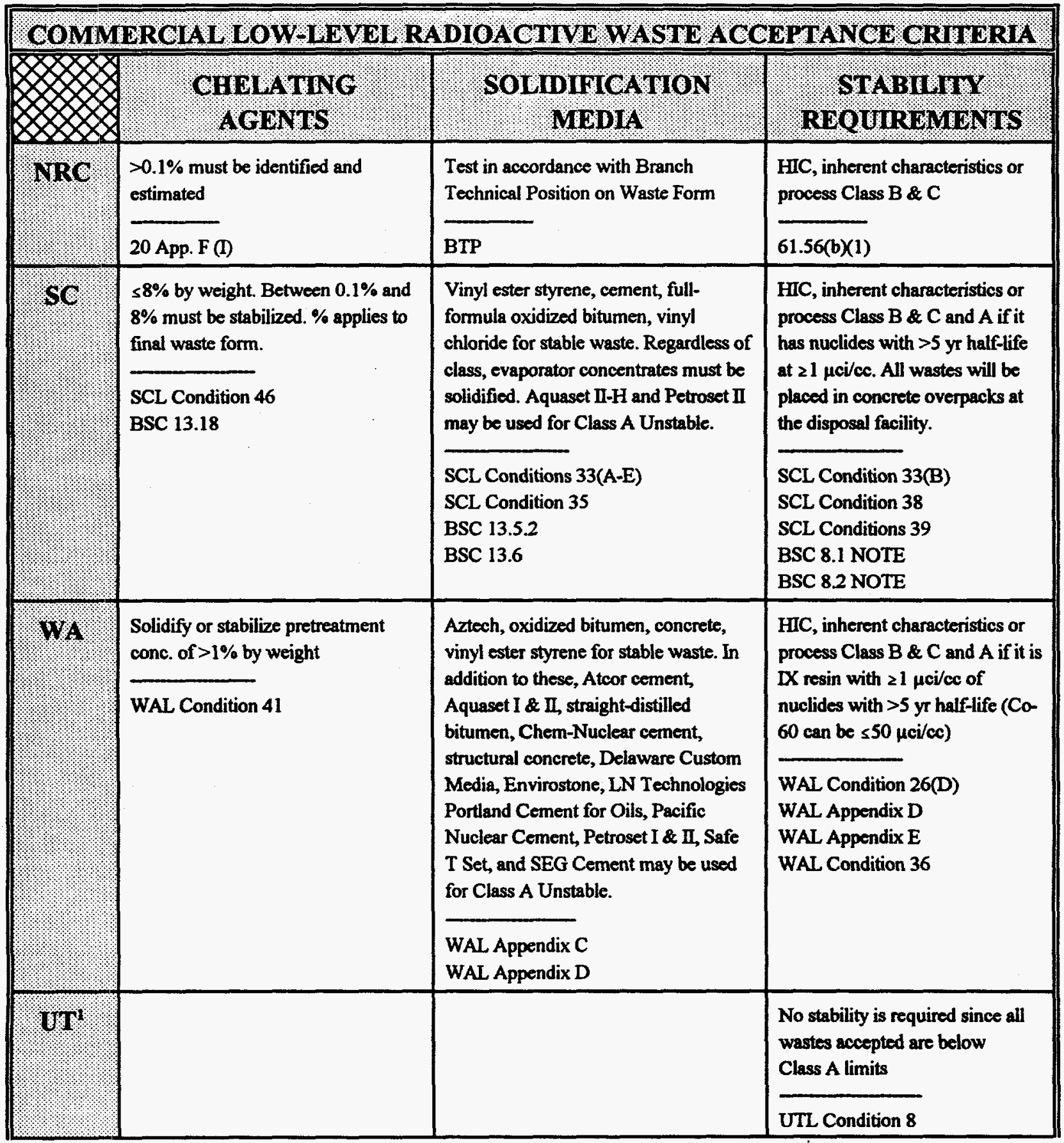




\begin{tabular}{|c|c|c|c|}
\hline $\mathbb{X} \geq$ & $\begin{array}{l}\text { CHELATING } \\
\text { AGENT }\end{array}$ & SOLDIFICA & REOUIRMMLYIS \\
\hline $\mathrm{MV}^{2}$ & $\frac{\text { Stabilize }>1 \% \text { by weight }}{\text { Condition } 40}$ & $\begin{array}{l}\text { Aztech, oxidized bitumen, Chem- } \\
\text { Nuclear cement, concrete, vinyl ester } \\
\text { styrene, LN Technologies cement, } \\
\text { Stock Equipment cement, } \\
\text { Westinghouse-Hittman cement for } \\
\text { stable waste. In addition, Aquaset I \& } \\
\text { II, straight-distilled bitumen, structural } \\
\text { concrete, Delaware Custom media, } \\
\text { Envirostone, Hittman Grout, Petroset I } \\
\text { \& II, and Safe T Set for unstable } \\
\text { waste. } \\
\text { Appendix C } \\
\text { Appendix D }\end{array}$ & $\begin{array}{l}\text { HIC, inherent characteristics or } \\
\text { process Class B \& C } \\
\text { Condition 22(c) } \\
\text { Condition } 25 \\
\text { Appendix D }\end{array}$ \\
\hline $9 \mathrm{~A}^{3}$ & $\begin{array}{l}>0.1 \% \text { pretreatment conc. must be } \\
\text { solidified or stabilized } \\
\text { Condition } 69\end{array}$ & $\begin{array}{l}\text { Aztech, oxidized bitumen, Chem- } \\
\text { Nuclear cement, concrete, vinyl ester } \\
\text { styrene, LN Technologies cement, } \\
\text { Stock Equipment cement, } \\
\text { Westinghouse-Hittman cement for } \\
\text { stable waste. In addition, Aquaset I \& } \\
\text { II, straight-distilled bitumen, structural } \\
\text { concrete, Delaware Custom media, } \\
\text { Envirostone, Hittman Grout, Petroset I } \\
\text { \& II, and Safe T Set for unstable } \\
\text { waste. } \\
\text { Appendix D } \\
\text { Appendix E }\end{array}$ & $\begin{array}{l}\text { HIC, inherent stability or process } \\
\text { Class B \& C and Class A if } \\
>30 \mathrm{R} / \mathrm{hr} . \\
\text { Condition } 58\end{array}$ \\
\hline$\pi$ & $\frac{s 8 \% \text { by weight }}{607.30(f)}$ & & $\frac{H I C \text { or process Class B \& C }}{607.30(b)}$ \\
\hline $\mathrm{Tx}^{\prime}$ & $\frac{s 8 \% \text { by weight }}{451.25(\mathrm{e})}$ & & $\begin{array}{l}\text { HC, inherent characteristics or } \\
\text { process Class B \& C } \\
451.26(\mathrm{a})\end{array}$ \\
\hline $\mathrm{PA}^{4}$ & $\frac{>0.1 \% \text { by weight must be identified }}{236.532}$ & & $\begin{array}{l}\text { HIC, inherent characteristics, or } \\
\text { processing to a stable form } \\
236.522(\mathrm{a}) \\
236.522(\mathrm{~b})\end{array}$ \\
\hline $\mathrm{NY}^{4}$ & & & $\begin{array}{l}\text { HIC, inherent characteristics, or } \\
\text { processing to a stable form } \\
382.81(\mathrm{~b})(1)\end{array}$ \\
\hline
\end{tabular}




\begin{tabular}{|c|c|c|c|}
\hline \\
\hline \multicolumn{4}{|c|}{ 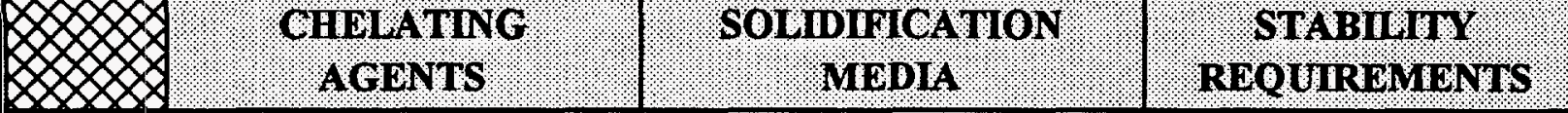 } \\
\hline \multicolumn{4}{|c|}{ 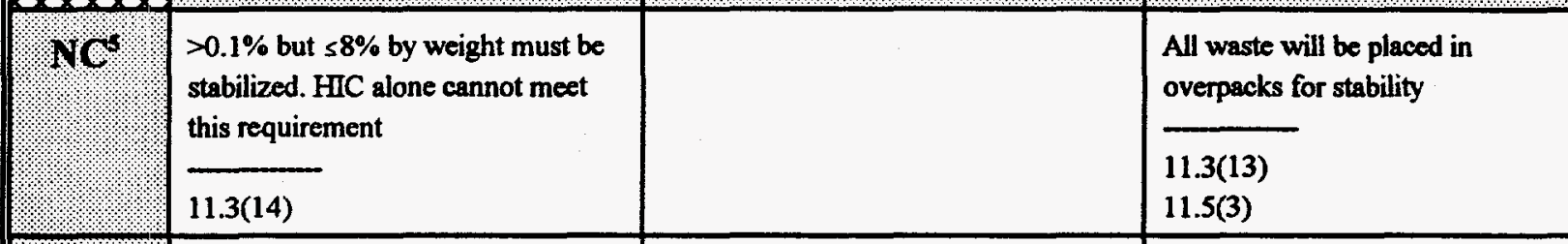 } \\
\hline $\mathrm{NO}^{\mathrm{S}}$ & $\begin{array}{l}\text { Solidify or stabilize }>0.1 \% \\
\text { pretreatment concentration. } \\
8.26\end{array}$ & $\begin{array}{l}\text { Aztech, oxidized bitumen, concrete, } \\
\text { vinyl ester styrene for stable waste. In } \\
\text { addition to these, Atcor cement, } \\
\text { Aquaset I \& II, straight-distilled } \\
\text { bitumen, Chem-Nuclear cement, } \\
\text { structural concrete, Delaware Custom } \\
\text { Media, Envirostone, Pacific Nuclear } \\
\text { Portland Cement, Petroset I \& II, Safe } \\
\text { T Set, and SEG Cement may be used } \\
\text { for Class A Unstable. } \\
\text { Appendix B } \\
\text { Appendix C }\end{array}$ & $\begin{array}{l}\text { In accordance with NRC BTP } \\
\text { and vendor topical reports. } \\
8.14 \\
9.3 \\
\text { Appendix C } \\
\text { Appendix D }\end{array}$ \\
\hline $\mathrm{MWW}^{6}$ & $\frac{s 8 \% \text { by weight after treatment. }}{\mathrm{B}(14)}$ & $\begin{array}{l}\text { Must meet requirements of NRC BTP } \\
\text { on Waste Form. } \\
\mathrm{B}(16)\end{array}$ & $\begin{array}{l}\text { Approved stabilization medium or } \\
\text { HIC. } \\
\mathrm{B}(2)\end{array}$ \\
\hline
\end{tabular}


Waste Acceptance Criteria:

\author{
Sorptive Media \\ Oil \\ Gas
}




\begin{tabular}{|c|c|c|c|}
\hline \multicolumn{4}{|c|}{ COMMERCIAL LOW LEVEL RADIOACTIVE WASTE A CCEPTANCE CRIIERIA } \\
\hline \pm 8 & SORPTIVE MEDIA & OL. & CHs \\
\hline NRP & $\frac{\text { Allowable }}{61.56(a)(2)}$ & & $\begin{array}{l}\leq 1.5 \mathrm{~atm} . \text { at } 20^{\circ} \mathrm{C} \text { and } \max . \\
\frac{100 \text { curies }}{61.56(\mathrm{a})(7)}\end{array}$ \\
\hline $\mathrm{SC}$ & $\begin{array}{l}\begin{array}{l}\text { Use only for incidental and } \\
\text { unintentional liquid in otherwise dry } \\
\text { solids. }\end{array} \\
\text { SCL Condition } 32(A) \\
\text { SCL Condition } 34\end{array}$ & $\begin{array}{l}\leq 1 \% \text { vol. trace and unintentional } \\
\text { (absorbed) } \\
\text { SCL Condition } 52 \\
\text { BSC } 13.8\end{array}$ & $\begin{array}{l}\mathrm{Kr}-85 \text { and } \mathrm{Xe-133} \text { at } \leq 1.5 \mathrm{~atm} \text {. } \\
\text { and max. } 100 \text { curies. Sealed } \\
\text { tritium gas sources ( } \leq 1.5 \mathrm{~atm} \text {.) at } \\
\leq 1,000 \text { curies per disposal } \\
\text { container. } \\
\text { SCL Condition } 47 \\
\text { BSC } 13.3\end{array}$ \\
\hline W\% & $\begin{array}{l}\text { May be used for liquids and listed in } \\
\text { Appendix G of license WN-I019-2, } \\
\text { Amendment } 18 \text {. Includes many } \\
\text { clays, diatomaceous earths, perlites, } \\
\text { and others } \\
\text { WAL Appendix G }\end{array}$ & $\begin{array}{l}\text { Solidify or stabilize pretreatment conc. } \\
\text { of }>10 \% \text { by weight } \\
\text { WAL Condition } 40\end{array}$ & $\begin{array}{l}\text { s1.5 atm. at } 20^{\circ} \mathrm{C} \text { and } \max . \\
100 \text { curies } \\
\text { WAL Condition } 35\end{array}$ \\
\hline \multicolumn{4}{|l|}{101} \\
\hline $\mathrm{N} \mathrm{V}^{2}$ & $\begin{array}{l}\text { May be used for liquids and listed in } \\
\text { Appendix E of license 13-11-0043- } \\
02 \text {, Amendment 13. Includes many } \\
\text { clays, perlites, diatomaceous carths, } \\
\text { and others } \\
\text { Appendix E }\end{array}$ & $\begin{array}{l}\text { Solidify or stabilize pretreatment cone. } \\
\text { of }>10 \% \text { by weight } \\
\text { Condition } 35\end{array}$ & $\begin{array}{l}\leq 1.5 \mathrm{~atm} . \text { at } 20^{\circ} \mathrm{C} \text { and } \max . \\
100 \text { curies } \\
\text { Condition } 30\end{array}$ \\
\hline $8 \mathrm{~A}$ & $\begin{array}{l}\text { Use only for incidental and } \\
\text { unintentional liquid in otherwise dry } \\
\text { solids } \\
\text { Condition } 55 \\
\text { Appendix C }\end{array}$ & $\frac{s 1 \% \text { vol. trace and unintentional }}{\text { Condition } 68}$ & $\begin{array}{l}\leq 1.5 \mathrm{~atm} . \text { at } 20^{\circ} \mathrm{C} \text { and } \max . \\
100 \text { curies. Tritium no more than } \\
100 \text { curies - must be special } \\
\text { packaged. } \\
\text { Condition } 65 \\
\text { Condition } 70\end{array}$ \\
\hline net & $\begin{array}{l}\text { Only for atmospheric condensation. } \\
\text { Cannot be used in lieu of } \\
\text { solidification to meet "no liquid" } \\
\text { requirement } \\
607.30(a)\end{array}$ & & \\
\hline $1 \times x$ & $\frac{\text { Allowable, but types not specified }}{451.26(c)}$ & $\frac{\leq 1 \% \mathrm{vol} . \text { trace }}{451.26(\mathrm{~g})}$ & $\begin{array}{l}\leq 1.5 \mathrm{~atm} . \text { at } 20^{\circ} \mathrm{C} \text { and } \max . \\
\frac{100 \text { curies }}{451.26(\mathrm{e})(4)}\end{array}$ \\
\hline
\end{tabular}




\begin{tabular}{|c|c|c|c|}
\hline \multicolumn{4}{|c|}{ COMMERCIAU LOW-LEVEL RADIOACTIVE WASTEACCEPTANCE CRITERA } \\
\hline 888 & SORPTIYE MEDLIA & OH. & OAS \\
\hline $\mathrm{PA}^{\prime}$ & $\frac{\text { Not acceptable }}{236.521(3)}$ & & $\begin{array}{l}<1.5 \text { atm. at } 20^{\circ} \mathrm{C} \text { and } \max . \\
\frac{100 \text { curies }}{236.521(8)}\end{array}$ \\
\hline NT. & $\frac{\text { Not acceptable }}{382.81(a)(2)}$ & $\frac{50.5 \% \text { vol. trace }}{383-8.3(a)(4)}$ & $\begin{array}{l}\leq 1.5 \mathrm{~atm} . \text { at } 20^{\circ} \mathrm{C} \text { and } \max . \\
100 \text { curies } \\
382.81(\mathrm{a})(7)\end{array}$ \\
\hline tres? & $\begin{array}{l}\text { Use only for incidental and } \\
\text { unintentional liquid in otherwise dry } \\
\text { solids. } \\
11.3(2)\end{array}$ & $\frac{51 \% \text { vol. trace }}{11.3(11)}$ & \\
\hline $\mathbb{N I}^{\prime}$ & $\begin{array}{l}\text { May be used for liquids with } \\
\text { approval; otherwise may only be } \\
\text { used for incidental free standing } \\
\text { liquid. Approved sorbents listed in } \\
\text { Appendix E. } \\
8.17 \\
\text { Appendix E }\end{array}$ & $\begin{array}{l}\text { Solidify or stabilize pretreatment conc. } \\
\text { of }>10 \% \text { by weight } \\
8.25\end{array}$ & $\begin{array}{l}\leq 1.5 \mathrm{~atm} . \text { at } 20^{\circ} \mathrm{C} \text { and } \max . \\
100 \text { curies } \\
8.21\end{array}$ \\
\hline $\mathrm{MW}$ & $\begin{array}{l}\text { Not acceptable except in packages } \\
\text { of radioactive consumer products } \\
\text { B (9) } \\
\text { C (9) }\end{array}$ & $\begin{array}{l}<1 \% \text { vol. incidental before } \\
\text { solidification. } \\
\mathrm{B}(15)\end{array}$ & $\begin{array}{l}\leq 1.5 \mathrm{~atm} . \text { at } 20^{\circ} \mathrm{C} \text { and } \max . \\
100 \text { curies of noble gases } \\
B(7)\end{array}$ \\
\hline
\end{tabular}




\title{
Waste Acceptance Criteria:
}

\author{
Biological Waste \\ Pyrophorics \\ Source Material
}




\begin{tabular}{|c|c|c|c|}
\hline \multicolumn{4}{|c|}{ COMMERCIAL LOW I EVEL RADIOACTIVE WASTE ACCEPTANC E CRIIIERIA } \\
\hline 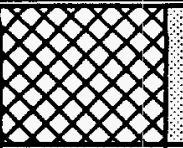 & : & PYROPHORICS, ? & 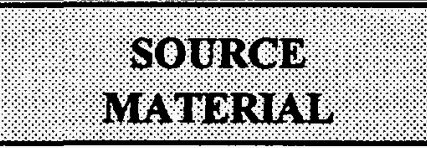 \\
\hline $\mathrm{NRC}$ & $\begin{array}{l}\text { Must be treated to reduce biological, } \\
\text { pathogenic, or infectious hazards. } \\
61.56(a)(8)\end{array}$ & $\frac{\text { Treat or package to be nonflammable }}{61.56(\mathrm{a})(6)}$ & \\
\hline $\mathrm{SO}$ & $\begin{array}{l}\text { Must be in certified } 17 \mathrm{H} \text { DOT } \\
\text { package with 4-mil. liner layered } \\
\text { with slaked lime and ag grade } 4 \\
\text { vermiculite or medium grade } \\
\text { diatomaceous earth (ratio } 30: 1: 10 \text { ) } \\
\text { and double packaged with at least } \\
4 \text { in. of absorbent in bottom of } \\
\text { outside container and absorbent } \\
\text { surrounding the upright inner } \\
\text { container. Must have standard ring } \\
\text { and bolt closure on inner container. } \\
\text { SCL Condition } 53 \\
\text { BSC } 13.2\end{array}$ & $\begin{array}{l}\text { Must be treated or packaged to be } \\
\text { nonflammable } \\
\text { SCL Condition } 51 \\
\text { BSC } 13.9\end{array}$ & $\begin{array}{l}60,000 \mathrm{lb} \\
\text { SCL Condition 5(B) } \\
\text { SCL Condition 6(B) } \\
\text { SCL Condition 7(B) }\end{array}$ \\
\hline WA & $\begin{array}{l}\text { Must be in 4-mil. liner layered with } \\
\text { absorbent (excluding perlites) and } \\
\text { lime. Must be double packaged with } \\
\text { absorbent in outside container. } \\
\text { WAL Condition } 33 \\
\text { WAL Condition } 34\end{array}$ & $\begin{array}{l}\text { Cannot react violently with water, } \\
\text { moisture or agitation } \\
\text { WAL Condition } 23\end{array}$ & $\begin{array}{l}36,000 \mathrm{~kg} \text {. Will also accept large } \\
\text { volume NORM of } \leq 0.002 \mu \mathrm{ci} / \mathrm{cc} \\
\text { WAL Condition } 6(\mathrm{~B}) \\
\text { WAL Condition } 7(\mathrm{~B}) \\
\text { WAL Condition } 8(\mathrm{~B}) \\
\text { WA Admin. Code } 246-249-080 \\
\text { for NORM }\end{array}$ \\
\hline \multicolumn{4}{|l|}{ UTt } \\
\hline $\mathrm{HV}^{2}$ & $\begin{array}{l}\text { Must be in 4-mil. liner layered with } \\
\text { absorbent and lime. Must be double } \\
\text { packaged with absorbent in outside } \\
\text { container. } \\
\text { Condition } 28 \\
\text { Condition } 29\end{array}$ & $\begin{array}{l}\text { Cannot react violently with water, } \\
\text { moisture or agitation } \\
\text { Condition } 20\end{array}$ & $\begin{array}{l}36,000 \mathrm{~kg} . \\
\text { Condition } 6(\mathrm{~B}) \\
\text { Condition 7(B) } \\
\text { Condition 8(B) }\end{array}$ \\
\hline $\mathrm{CA}^{3}$ & $\begin{array}{l}\text { Must be in 4-mil. liner layered with } \\
\text { slaked lime and medium grade } \\
\text { diatomaceous earth (ratio 30:1:10) } \\
\text { and double packaged with } \\
\text { absorbent in outside container. } \\
\text { Condition } 61 \\
\text { Condition } 62\end{array}$ & $\begin{array}{l}\text { Cannot react violently with water, } \\
\text { moisture or agitation } \\
\text { Condition } 54\end{array}$ & $\begin{array}{l}36,000 \mathrm{~kg} \\
\text { Condition } 6(\mathrm{~b}) \\
\text { Condition } 7(\mathrm{~b}) \\
\text { Condition } 8(\mathrm{~b})\end{array}$ \\
\hline
\end{tabular}




\begin{tabular}{|c|c|c|c|}
\hline 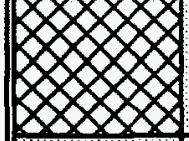 & $\begin{array}{l}\text { BIOLOGICAL, } \\
\text { W/ASE }\end{array}$ & PYROPHORICS & : $:$ : $:$ : $\mathrm{MATHRT}$ \\
\hline $\mathrm{H}$ & $\begin{array}{l}\text { Must be treated to reduce biological, } \\
\text { pathogenic, or infectious hazards. } \\
\frac{607.30(\mathrm{~h})}{}\end{array}$ & $\begin{array}{l}\text { Must be treated to eliminate } \\
\text { pyrophoric hazard. } \\
607.30(\mathrm{~g})\end{array}$ & \\
\hline 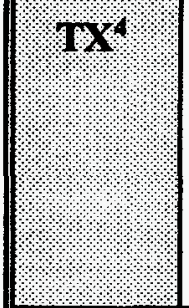 & $\begin{array}{l}\text { Double pkg. with absorbent. Must } \\
\text { be layered with slaked lime and } \\
\text { medium grade diatomaceous earth } \\
\text { (ratio 30:1:10) } \\
\begin{array}{l}451.25(\mathrm{f}) \\
451.26(\mathrm{f})\end{array}\end{array}$ & $\begin{array}{l}\text { Must be treated or packaged to be } \\
\text { nonflammable } \\
\frac{451.26 \text { (d) }}{}\end{array}$ & \\
\hline $\mathrm{P}+1$ & $\begin{array}{l}\text { Must be treated to reduce potential } \\
\text { hazard from nonradiological } \\
\text { materials } \\
236.521(9)\end{array}$ & $\begin{array}{l}\text { Must be treated and packaged to be } \\
\text { nonflammable } \\
236.521(7)\end{array}$ & \\
\hline $\mathrm{N}$ & $\begin{array}{l}\text { Must be treated to reduce potential } \\
\text { hazard from nonradiological } \\
\text { materials. Animal carcasses human } \\
\text { tissue, and petroleum-based liquids } \\
\text { must be incinerated or stabilized. } \\
382.81 \text { (a)(8) } \\
383.8 .3(\mathrm{a})\end{array}$ & $\begin{array}{l}\text { Must be treated and packaged to be } \\
\text { nonflammable } \\
382.81(a)(6)\end{array}$ & $\begin{array}{l}\text { NARM allowed after review } \\
383-8.10 \\
382.83\end{array}$ \\
\hline $\mathrm{NCO}^{\circ}$ & & $\begin{array}{l}\text { Final waste form cannot be pyrophoric } \\
\text { or flammable } \\
11.3(11)\end{array}$ & \\
\hline $\mathrm{NL}^{\mathrm{S}}$ & $\begin{array}{l}\text { Animal carcasses must be in 4-mil. } \\
\text { liner layered with absorbent } \\
\text { (excluding perlites) and lime (ratio } \\
10: 1) \text {. Must be double packaged } \\
\text { (DOT 7A or 17H) with absorbent in } \\
\text { outside container. Other biological } \\
\text { waste must be treated to reduce the } \\
\text { hazard and packaged similarly. } \\
8.19 \\
8.20\end{array}$ & $\begin{array}{l}\text { Final waste form must be treated, } \\
\text { prepared, and packaged to be } \\
\text { nonflammable. } \\
8.8\end{array}$ & \\
\hline
\end{tabular}




\begin{tabular}{|c|c|c|c|}
\hline \multicolumn{4}{|c|}{ COMMERCIAL LOW-LEVEL RADIOACTIVE WASTE ACCEPTANCE CRIILRIA } \\
\hline XXX & $\begin{array}{l}\text { BIOLOGIOAL } \\
\text { WASTE }\end{array}$ & PYROPHORICS & $\begin{array}{l}\text { SOURCE } \\
\text { MATERIAI }\end{array}$ \\
\hline $10 W^{6}$ & $\begin{array}{l}\text { Biological, pathogenic, and } \\
\text { infectious wastes must be treated to } \\
\text { reduce potential hazard from } \\
\text { nonradiological materials. } \\
\text { Untreated animal carcasses and } \\
\text { biowaste capable of generating gas } \\
\text { or fumes are not acceptable. Prior } \\
\text { practice of packaging carcasses in } \\
\text { poly-liners, double steel drums, } \\
\text { lime, and absorbent will not be } \\
\text { permitted as final waste form. } \\
\text { B (6) } \\
\text { C (6) }\end{array}$ & $\begin{array}{l}\text { Cannot react violently with water, } \\
\text { moisture, or agitation. } \\
\mathrm{B}(3)\end{array}$ & \\
\hline
\end{tabular}


Waste Acceptance Criteria:

\author{
Special Nuclear Material \\ Package Dimensions \\ Incinerator Ash
}




\begin{tabular}{|c|c|c|c|}
\hline 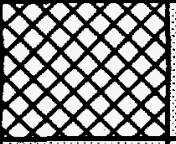 & SPRPRTHOCHAR & DAMEAGE & 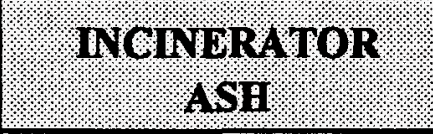 \\
\hline \multicolumn{4}{|l|}{$\mathrm{NRC}$} \\
\hline $\mathrm{SC}$ & $\begin{array}{l}200 \mathrm{~g} \mathrm{U}-233 \text { or } 350 \mathrm{~g} \mathrm{U}-235 \mathrm{max} . \\
\text { in a package. } \\
\text { NRCL Condition } 1 \\
\text { SCL Condition } 40 \\
\text { BSC } 13.10\end{array}$ & $\begin{array}{l}\text { No dimensions are specified in the } \\
\text { license although all classes will be } \\
\text { placed in concrete overpacks called } \\
\text { vaults at the disposal facility. } \\
\text { Dimensions in BSC are max. box size } \\
\text { of } 9 \mathrm{ft} 4 \text { in. } L \times 7 \mathrm{ft} 6 \text { in. W } \times 9 \mathrm{ft} 2 \mathrm{in} \text {. } \\
\mathrm{H} \text { with max. weight of } 54,000 \mathrm{lb} \text {. } \\
\text { SCL Condition } 39 \\
\text { BSC 7.5 } \\
\text { BSC 8.11 } \\
\text { BSC 9.3.1 }\end{array}$ & $\begin{array}{l}\text { Must solidify, treat or package } \\
\text { (with binding matrix) to be } \\
\text { nondispersible } \\
\text { SCL Condition } 43 \\
\text { SCL Condition } 45 \\
\text { BSC 13.7.1.2 }\end{array}$ \\
\hline$w_{H}$ & $\begin{array}{l}60 \mathrm{~g} \mathrm{U}-233 \text { or } 100 \mathrm{~g} \mathrm{U}-235 \text { or } 60 \\
\text { grams plutonium max. in a package } \\
\text { NRCL } 5(a)-(c) \\
\text { NRCL } 6\end{array}$ & & $\begin{array}{l}\text { Must be solidified, granular or } \\
\text { treated to be nondispersible in air } \\
\text { WAL Condition } 39\end{array}$ \\
\hline $101 \%$ & $\begin{array}{l}\begin{array}{l}350 \mathrm{~g} \mathrm{U}-235 \text { or } 200 \mathrm{~g} \mathrm{U}-233 \text { or } \\
200 \mathrm{~g} \mathrm{Pu}\end{array} \\
\text { UTL Condition } 13\end{array}$ & & \\
\hline $\mathrm{YW}^{2}$. & $\begin{array}{l}200 \mathrm{~g} \text { U-233 or } 350 \mathrm{~g} \mathrm{U}-235 \text { or } \\
200 \mathrm{~g} \text { plutonium max. per package } \\
\text { Condition } 6(\mathrm{C}) \\
\text { Condition } 7(\mathrm{C}) \\
\text { Condition } 8(\mathrm{C})\end{array}$ & & $\begin{array}{l}\text { Must be solidified, granular or } \\
\text { treated to be nondispersible in air } \\
\text { Condition } 33\end{array}$ \\
\hline$e^{3}$ & $\begin{array}{l}500 \mathrm{~g} \mathrm{U}-235 \text { or } 300 \mathrm{~g} \mathrm{U}-233 \text { or } \\
300 \mathrm{~g} \text { plutonium max. per shipment } \\
\text { Note to Condition 8(c) }\end{array}$ & & $\begin{array}{l}\text { Must be treated to be } \\
\text { nondispersible in air } \\
\text { Condition } 67\end{array}$ \\
\hline $11 \%$ & & & $\frac{\text { Must be solidified }}{607.30(a)(2)}$ \\
\hline 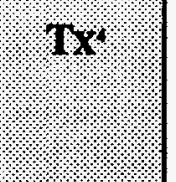 & & $\begin{array}{l}\text { Must fit in } 81 \text { in. ht. and } 80 \text { in. dia. } \\
\text { cylinder } \\
451.27\end{array}$ & $\frac{\text { Must be nondispersible in air }}{451.26(\mathrm{~h})}$ \\
\hline $\mathrm{PA}$ & & & \\
\hline
\end{tabular}




\begin{tabular}{|c|c|c|c|}
\hline \multicolumn{4}{|c|}{ COMMERCIAL LOW LEVEL RADIOACTIVE WASTE ACCEPTANCE CRIYYERIA. } \\
\hline 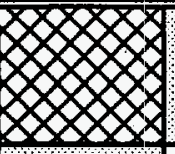 & I: & PIMAKSTER & 1. \\
\hline $14^{1}$ & $\begin{array}{l}350 \mathrm{~g} \mathrm{U}-235 \text { or } 200 \mathrm{~g} \mathrm{U}-233 \text { or } \\
200 \mathrm{~g} \mathrm{Pu} \\
383-8.6(\mathrm{a})(5)\end{array}$ & & \\
\hline \multicolumn{4}{|l|}{$\mathrm{NC}^{5}$} \\
\hline $\mathrm{NO}^{6}$. & & $\begin{array}{l}\text { Minimum size: } 55 \text {-gal. drum. Class B } \\
\text { or } \mathrm{C}(<1,000 \mathrm{R} / \mathrm{hr}) \text { must be } \leq 6 \mathrm{ft} 6 \mathrm{in} \text {. } \\
\text { height and } 6 \mathrm{ft} 3 \mathrm{in} \text {. diameter. Class } \mathrm{B} \\
\text { or } \mathrm{C}(>1,000 \mathrm{R} / \mathrm{hr}) \text { must be } \leq 9 \mathrm{ft} 2 \mathrm{in} \text {. } \\
\text { height and } 3 \mathrm{ft} 0 \text { in. diameter (max } \\
\text { sum of liner and cask } \leq 23 \mathrm{ft} 5 \mathrm{in} \text {.). } \\
\text { Maximum weight } 30,000 \mathrm{lb} \text {. } \\
\text { Maximum contact dose rate } \\
30,000 \mathrm{R} / \mathrm{hr} \text {. } \\
8.3 .2 \\
12.2 \\
12.3 \\
12.4 \\
12.5 \\
13.2\end{array}$ & $\begin{array}{l}\text { Must be treated to be } \\
\text { nondispersible in air, exclusive of } \\
\text { packaging. Class B and C ash } \\
\text { must also be stabilized. } \\
8.24 \\
9.3\end{array}$ \\
\hline MWro & & $\begin{array}{l}\text { Discouraged from using extremely } \\
\text { large or odd-shaped containers } \\
C(4)\end{array}$ & $\begin{array}{l}\text { Dry powders and ash must be } \\
\text { treated to be nondispersible in air. } \\
\text { B(5) }\end{array}$ \\
\hline
\end{tabular}


Waste Acceptance Criteria:

\author{
Dewatered Resin \\ Transuranics \\ Mixed Waste
}




\begin{tabular}{|c|c|c|c|}
\hline \multicolumn{4}{|c|}{ COMMIBRCLAL LOW-LEVEL RADIOACTIVE WASTE A CCEPTANCE ORIBGRIA } \\
\hline XXXX & :1: DEWATURDD & TRANSURAMIOS & 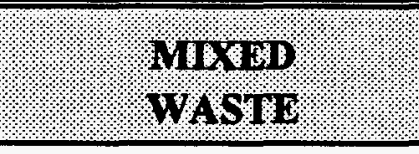 \\
\hline NRO & $\begin{array}{l}\frac{\text { Accepted }}{61.56(\mathrm{a})(3)} \\
61.56(\mathrm{~b})(2) \\
\text { BTP Section C.3 and C.4 }\end{array}$ & & \\
\hline SC. & $\begin{array}{l}\text { Accepted } \\
\text { SCL Condition } 36 \\
\text { SCL Condition } 37 \\
\text { SCL Condition } 38 \\
\text { BSC } 13.16\end{array}$ & $\begin{array}{l}\text { Activity must be evenly distributed and } \\
\text { incidental }(\leq 1 \%) \text {. Accepts whole } \\
\text { smoke detectors with Am-241 foils. } \\
\text { SCL Condition } 40 \\
\text { SCL Condition } 41 \\
\text { BSC } 13.10 .2 \text { for Pu } \\
\text { BSC } 13.12\end{array}$ & $\begin{array}{l}\text { Not accepted. Nonradioactively } \\
\text { contaminated lead used for } \\
\text { shielding may be accepted. No } \\
\text { PCB allowed. Asbestos may be } \\
\text { accepted. } \\
\text { SCL Condition } 48 \\
\text { BSC } 13.19 \\
\text { BSC } 13.20 \\
\text { BSC } 13.21 \\
\text { BSC } 13.22\end{array}$ \\
\hline W\% & $\begin{array}{l}\text { Accepted } \\
\text { WAL Condition } 28 \\
\text { WAL Condition } 29 \\
\text { WAL Condition } 36\end{array}$ & $\begin{array}{l}\text { Activity must be evenly distributed. } \\
\text { Accepts whole smoke detectors and } \\
\text { exempt consumer products. } \\
\text { WAL Condition } 37 \\
\text { WAL Condition } 38\end{array}$ & \\
\hline U1'. & $\begin{array}{l}\text { A special evaluation is required } \\
\text { before disposal. Ion exchange } \\
\text { resins will be mixed with soil at a } 9 \\
\text { parts soil:1 part resin ratio before } \\
\text { emplacement } \\
\text { LTR-08/95 } \\
\text { LTR-09/95 } \\
\text { UT IN-05-96 }\end{array}$ & & $\begin{array}{l}\text { Certain mixed wastes are } \\
\text { acceptable. } \\
\text { UTL Condition } 15 \\
\text { UTHWP }\end{array}$ \\
\hline $\mathrm{rv}$ & $\frac{\text { Accepted }}{\text { Condition } 24}$ & $\begin{array}{l}\text { Accepted whole smoke detectors and } \\
\text { exempt consumer products } \\
\text { Condition } 32\end{array}$ & . \\
\hline $\mathrm{CA}^{3}$ & $\frac{\text { Not accepted }}{\text { Condition } 59}$ & $\begin{array}{l}\text { Accepts whole smoke detectors and } \\
\text { exempt consumer products } \\
\text { Condition } 66\end{array}$ & \\
\hline $1 \%$ & $\frac{\text { Not accepted }}{607.30(a)(3)}$ & & \\
\hline $1 \%$ & & & \\
\hline
\end{tabular}




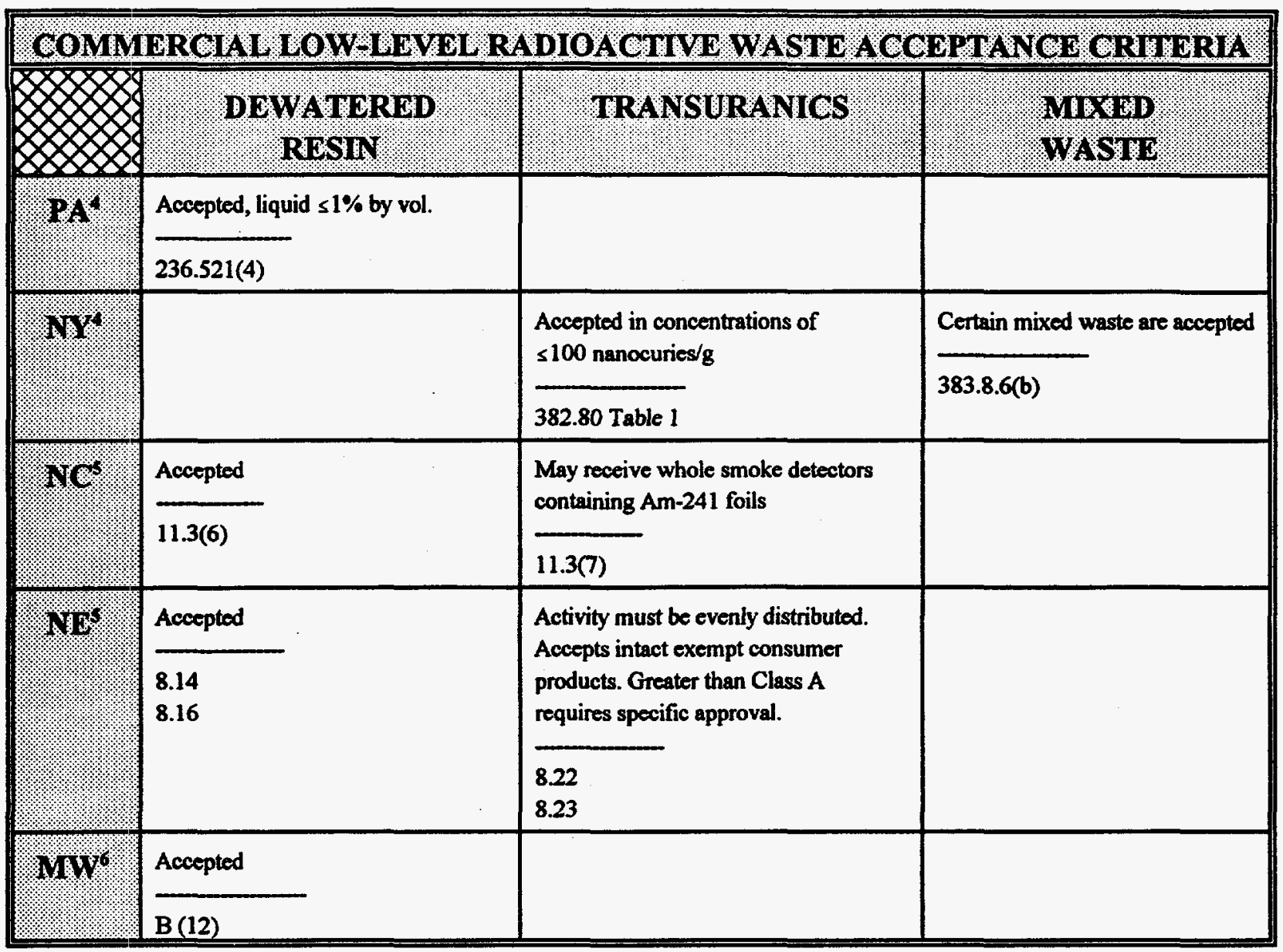

Notes:

1. Utah license is restricted to less than Class A limits for most radionuclides.

2. Nevada WAC are no longer in effect.

3. California license has been issued and has withstood all legal challenges and appeais.

4. These are proposed or promulgated criteria developed by a state agency. Final WAC must be approved by the appropriate regulatory agency through guidance, regulations, or license conditions.

5. These are proposed/suggested criteria developed by the license applicant (NC and NE).

6. Midurest compact commission criteria are preliminary. 